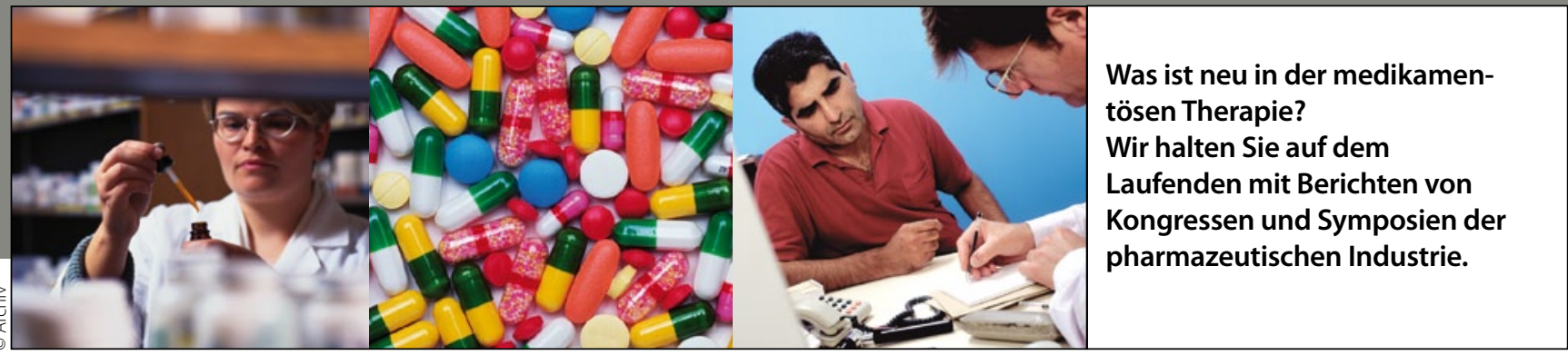

\title{
Hypertonie im Alter
}

\section{Individuell, behutsam und kombiniert behandeln!}

— Ein normaler Blutdruck ist bei Älteren die Ausnahme: $80 \%$ aller über 80 -Jährigen sind hyperton, und die meisten Patienten weisen im Alter einen isolierten systolischen Bluthochdruck auf, erklärte Prof. Reinhold Kreutz, Berlin.

Gemäß den Empfehlungen der Hochdruckliga macht sich eine Hypertoniebehandlung auch bei über 80-Jährigen prognostisch bezahlt. Der systolische Zielblutdruck liegt bis 80 Jahre bei $140 \mathrm{mmHg}$ und ab 80 Jahren bei $150 \mathrm{mmHg}$, so Kreutz. Empfohlen werden die gleichen Substanzklassen wie bei jüngeren Patienten, vorzugsweise in Kombinationen, um Nebenwirkungen zu minimieren.

Kreutz empfiehlt, im Alter sehr individualisiert und behutsam zu behandeln. Er verweist darauf, dass in wichtigen Meilensteinstudien wie SHEP (mit Chlorthalidon), Syst-Eur (mit Nifedipin), ASCOT oder ACCOMPLISH das Durchschnittsalter zwischen 60 und 70 Jahren lag und damit Evidenzen im Rentenalter vorliegen. ASCOT zeigte, dass die Kombination ACE-Hemmer

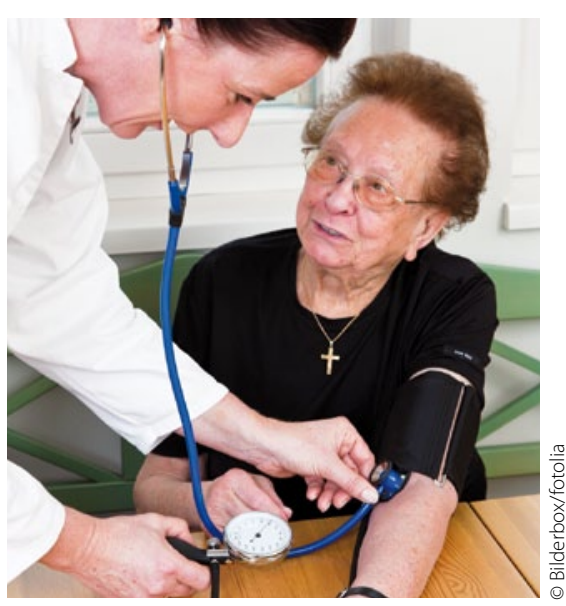

und Kalziumantagonist der Kombination Betablocker/Diuretika prognostisch überlegen ist. Laut ACCOMPLISH (Durchschnittsalter 68 Jahre) verhütet die Kombination RAS-Blocker/Kalziumantagonist mehr Komplikationen als die Kombination RAS-Blocker/Diuretikum.

$\mathrm{AT}_{1}$-Blocker, Kalziumantagonisten und ggf. Diuretikum zusätzlich können in Fixkombination verschrieben werden (Vocado ${ }^{\circledR}$, VocadoHCT $^{\circledR}$ ). Diese bewirken eine starke Blutdrucksenkung, sie erleichtern dem Patienten die Therapie und erhöhen die Adhärenz, ergänzte Dr. F. Mahfoud, Homburg/Saar. Eine gute Adhärenz, so Mahfoud, ist relevant für die Prognose. Speziell für die Fixkombination Olmesar-
tan/Amlodipin liegen Daten vor, denen zufolge die Kombination bei über 65-Jährigen nicht minder wirksam ist als bei unter 65-Jährigen, berichtete Kreutz.

In der Versorgungsrealität ist noch Raum für Verbesserungen: Wie eine Untersuchung von Verordnungen bei älteren Pflegeheimbewohnern zeigt, dominieren unter den Antihypertensiva Diuretika, v.a. Schleifendiuretika. Diese jedoch sind zur Hochdrucktherapie im Alter nicht gut geeignet, so Kreutz.

- Dr. med. Dirk Einecke

Quelle: Symposium „Intelligentes Hypertonie Management", Kongress der Deutschen Hochdruckliga, Berlin, Dezember 2012 (Veranstalter: Berlin-Chemie)

\section{Parodontitis steigert das Risiko für systemische Erkrankungen Risikopatienten zum Zahnarzt schicken}

\author{
— Etwa jeder zweite Erwachsene zwischen \\ 35 und 44 Jahren hat eine mittelschwere \\ Parodontitis, jeder fünfte eine schwere \\ Form der Parodontitis, so die Ergebnisse \\ der Vierten Deutschen Mundgesundheits- \\ studie. Die Entzündung des Zahnhalteap- \\ parats hat nicht nur für die Mundgesund- \\ heit weitreichende Folgen, sondern für die \\ Gesundheit des gesamten Körpers. Studi- \\ en zufolge steigert eine Parodontitis das \\ Risiko für \\ - Schlaganfall um das Siebenfache, \\ - Arthritis um das Sechsfache, \\ - Herzinfarkt um das Dreifache, \\ - Früh- und Fehlgeburten um das \\ 4,8-fache.
}

Den gefährlichen Wechselwirkungen kann eine möglichst frühzeitige Behandlung der Parodontitis vorbeugen. Doch nur wenige Betroffene befinden sich in Behandlung, vor allem weil die Paradontitis lange Zeit keinerlei Schmerzen verursacht und unbemerkt bleibt. Ein Zahnarztbesuch könnte sich daher lohnen: Mit dem Schnelltest PerioMarker ${ }^{\circledast}$ ist inzwischen eine Früherkennung der Parodontitis möglich. Damit lassen sich versteckte Entzündungen nachweisen, noch bevor klinische Zeichen wie lockere Zähne sichtbar werden.

\footnotetext{
- Dr. Judith Neumaier

Quelle: Nach Informationen der Firma Hager \& Werken
} 